\title{
O Papel da (Re)Duplicação na Expressão de Pluracionalidade em Libras
}

\author{
The Role of (Re)Duplication in the \\ Expression of Pluractionality in Libra
}

Luciana Sanchez-Mendes

Rimar Ramalho Segala*

André Nogueira Xavier ${ }^{* *+}$

Resumo

Este artigo objetiva mostrar diferentes formas de expressar pluracionalidade na língua brasileira de sinais (libras). Mais precisamente, ele foca em três recursos formais - a duplicação de mãos, a repetição simples de movimento e a repetição de movimento alternado - e seu uso na expressão de plural de argumentos, plural de eventos e intensidade na libras. Uma parte dos dados aqui discutidos provém da reanálise interpretativa, por um sinalizante surdo e nativo de libras, dos sinais analisados por Sanchez-Mendes e Xavier (2016). A outra parte provém da eliciação, do mesmo sinalizante, desses sinais, levando em conta não apenas o número de mãos, mas também a possibilidade de o realizar repetidamente em sequência e, no caso da forma bimanual, através de movimento alternado. Além disso, eliciou-se também a interpretação de cada uma dessas formas. Do ponto de vista morfofonológico, nossos resultados mostram que nem todas as formas são possíveis para todos os sinais eliciados. Já da perspectiva semântica, eles reforçam que todas as leituras aqui eliciadas podem ser capturadas pela noção de pluracionalidade e formalmente analisadas como uma operação sobre uma variável escalar que pode ser cardinalidade ou intensidade.

Palavras-chave: libras; pluracionalidade; operação de plural.

\footnotetext{
* Universidade Federal Fluminense

** Universidade Federal de São Carlos

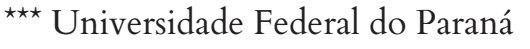




\begin{abstract}
This paper aims to show different ways to express pluractionality in Libras. More specifically, it focuses on three formal resources - hand doubling, simple movement repetition and alternating movement repetition - and their use in the expression of plurality of arguments, plurality of events and intensity. Part of the data discussed here come from an interpretation reanalysis of SanchezMendes and Xavier's (2016) data by a native Libras signer. The other part comes from their elicitation from the same signer, considering not only their number of hands, but also the possibility to produce them repeatedly in a row and, in the case of the two-handed form, with alternating movement. In addition, the interpretation of the readings of each form was also elicited. From the morphophonological viewpoint, our results show that not all forms are possible for all the signs elicited. From the semantic perspective, they reinforce that all their readings can be captured by the notion of pluractionality and can be formally analyzed as an operation over a scalar variable that can be cardinality or intensity.
\end{abstract}

Keywords: Libras; pluractionality; plural operation. 
L. SANCHEZ-

Mendes, R. R.

Segala \& A.

N. XAvier

O papel da (re)

duplicação na

expressão de

pluracionalidade

em libras

\section{Introdução}

$\mathrm{O}$ objetivo deste artigo é apresentar diferentes formas de se expressar pluracionalidade em libras, entendida como a marcação de plural nos verbos que não se confunde com morfemas de concordância, bem como argumentar em favor de uma análise semântica unificada que dê conta de todas leituras apresentadas por essa operação.

A primeira fase de nossa pesquisa, apesar de enfocar a duplicação de mãos do ponto de vista fonético-fonológico, evidenciou que esse processo pode se dar por diferentes fatores semânticos: expressão de pluralidade, aspecto e intensidade (XAVIER, 2014). Na segunda fase do estudo, Sanchez-Mendes e Xavier (2016) argumentaram que esses fatores semânticos podem ser capturados por uma única operação semântica chamada na literatura de pluracionalidade. Em seu estudo, os autores selecionaram e reanalisaram 14 verbos dos 28 sinais originalmente coletados e analisados por Xavier (2014). Na presente fase, avançamos na descrição e análise da expressão de pluracionalidade em libras (i) apresentando uma reanálise interpretativa dos dados de Xavier por um sinalizante nativo e (ii) explorando a interface entre a morfofonologia e a semântica, ao investigar a diferença entre a repetição de movimento simples versus a repetição de movimento alternado de verbos tipicamente produzidos com uma mão.
Revista Letras,

Curitiba, UfPr, n. 96, pp.487-508, jul./dez. 2017. ISSN 2236-0999 (versão eletrônica) 
Este artigo está organizado da seguinte forma: a seção 2, a seguir, aborda a pluracionalidade nas línguas orais; a seção 3 trata desse fenômeno em línguas de sinais, mais especificamente, ela sumariza os achados de SanchezMendes e Xavier (2016) sobre a libras e os de Kuhn e Aristodemo (2017) sobre a língua de sinais francesa, que embasaram a fase atual de nossa pesquisa, aqui reportada; a seção 4 descreve a metodologia adotada na coleta de dados; a seção 5 apresenta os resultados obtidos e a seção 6 apresenta a nossa proposta formal para a pluracionalidade baseada na operação semântica de plural e em variáveis sobre escalas.

\section{Pluracionalidade em Línguas Orais}

O objetivo desta seção é apresentar o fenômeno da pluracionalidade em línguas orais na sua variedade de formas e interpretações. A pluracionalidade é uma operação semântica expressa no sintagma verbal associada à pluralidade, distribuição, continuidade e intensidade e manifesta em uma variedade de línguas (CUSIC, 1981; LASERSOHN, 1995; CABREDO HOFHERR; LACA, 2012).

Do ponto de vista da forma, os marcadores pluracionais são frequentemente expressos por meio de reduplicação. Há muitas formas de repetição que são usualmente chamadas de reduplicação. Segundo Rubino (2005), a reduplicação diz respeito à repetição do material fonológico de uma palavra com propósitos semânticos ou gramaticais. A diferença entre mera repetição e reduplicação está relacionada à noção de palavra (GIL, 2005). A repetição é um fenômeno do nível da palavra, enquanto que a reduplicação está associada a um fenômeno morfológico.

A reduplicação pode ser parcial ou total; simples ou complexa'. Um exemplo de reduplicação parcial é a o marcador pluracional do sqwamish (salish). Em (1b), a repetição da forma 'tl'ex' (ganhar) indica que o evento expresso pelo verbo está distribuído no tempo.

Sqwamish (1) a. chen tl'exwenk.

1s.SG ganhar.INTR

'Eu ganhei.'

b. chen tl'ex-tl'exwenk.

1s.sG REDUP-ganhar.INTR

'Eu estou ganhando o tempo todo.'

(BAR-EL, 2008)

A reduplicação parcial pode ser em posição inicial, medial ou final. Em sqwamish, acima, temos um caso de reduplicação em posição inicial. Para

1 Há ainda um tipo de reduplicação automática que envolve a repetição acompanhada de um afixo. 
L. SANCHEZ-

Mendes, R. R.

Segala \& A.

N. XAVIER

O papel da (re)

duplicação na

expressão de

pluracionalidade

em libras

ilustrações de todos os casos, ver Rubino (2005). A reduplicação é total quando ocorre a repetição da palavra inteira ou da raiz inteira da palavra. Um exemplo de reduplicação total da raiz é da língua karitiana (tupi), em que há a repetição da raiz verbal 'mangat' (levantar). Observe que os morfemas flexionais de pessoa, modo e tempo não são repetidos.

(2) a. Inacio $\varnothing$-na-mangat- $\varnothing \quad$ Nadia ka'it.

Inacio 3-DECL-levantar-NFut Nadia hoje

'Inacio levantou Nadia hoje uma ou mais vezes.'

b. Inacio $\varnothing$-na-mangat-mangat- $\varnothing$ Nadia ka'it.

Inacio 3-DECL-levantar-NFUt Nadia hoje

'Inacio levantou Nadia hoje uma ou mais vezes.'

(MÜLLER, SANCHEZ-MENDES, 2010)

Ademais, a reduplicação pode ocorrer mais de uma vez. Veja abaixo um exemplo do mokilês (austronésia) em que é possível haver reduplicação uma ou duas vezes.
a. roar
'estremecer'
Mokilês
b. roarroar 'estar estremecendo'
c. roarroarroar 'continuar estremecendo'

(HARRISON, 1973 apud RUBINO, 2005)

A reduplicação pode ser simples e apresentar apenas repetição de palavra ou parte da palavra, como nos casos acima, ou ainda, pode ser complexa. Neste último caso, além da repetição, há também um acréscimo fonológico. Um exemplo de reduplicação complexa é o do yakan (austronésia) em que há uma repetição da consoante somada à sequência vocálica 'ew'.

$\begin{array}{llll}\text { (4) } & \text { labo } & \text { 'cair' } & \text { Yacan } \\ & \text { lewlabo } & \text { 'continuar caindo' } \\ \text { b. } & \text { duddag } & \text { 'diminuir' } \\ & \text { dewduddag } & \text { 'continuar diminuindo' }\end{array}$

(RUBINO, 2005)

Além da forma reduplicativa frequente, os marcadores pluracionais também podem se manifestar na forma de afixos ou de alternância vocálica (LASERSOHN, 1995). Por exemplo, em †hoan (khoisan), a inserção do prefixo 'kí-' e do sufixo '-qllo' expressam a noção de distribuição de eventos plurais no espaço.

Revista Letras,

Curitiba, UFPR, n. 96, pp.487-508, jul./dez. 2017. ISSN 2236-0999 (versão eletrônica) 
(5)

$$
\begin{array}{lll}
\text { Tsi } & \text { i } & \text { kí-’am-qllo. } \\
\text { 3PL } & \text { PAST } & \text { kí-comer-qllo }
\end{array}
$$

'Eles comeram por toda parte.'

(COLLINS, 2001)

Outra forma de se expressar pluracionalidade é a alternância vocálica. Os exemplos do yuma (yuman) com a alternância 'e' / 'a:' ilustram essa estratégia.

Yacan
(6) a. axwelyk 'ele morde'
b. axwa:lyk 'ele morde aqui e ali'

(LASERSOHN, 1995)

Além da variedade do ponto de vista da forma, a pluracionalidade está associada a uma gama de interpretações. Nesta seção, apresentaremos apenas as leituras de plural de argumentos, plural de eventos e de intensidade, por serem as mesmas que Sanchez-Mendes e Xavier (2016) identificaram na libras e que reportaremos na seção seguinte. Para um panorama geral de leituras atestadas da pluracionalidade, ver Lasersohn (1995).

Um exemplo de pluracionalidade expressando pluralização de argumentos ocorre em nukuoro (austronésia). Como se pode observar no exemplo em (7), a reduplicação parcial da consoante inicial indica uma pluralização dos participantes da ação.

492

Nukuoro

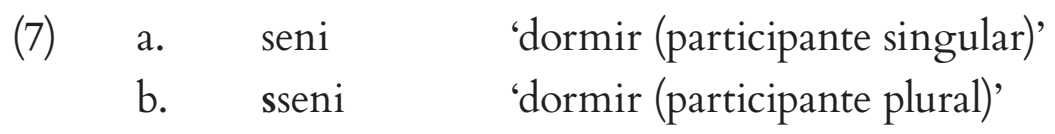

(RUBINO, 2005)

Uma língua que apresenta pluracionalidade na expressão de plural de eventos é o groenlandês do oeste (esquimó) (West Greenlandic). Nessa língua, o morfema 'qattaar' indica uma repetição dos eventos, conforme ilustrado em (8).

Groenlandês

(8)

$\begin{array}{lll}\text { Qaartartut } & \text { sivisuumik } & \text { qaaqattaarput. } \\ \text { qaartartu-t } & \text { sivisuu-mik } & \text { qaar-qattaar-put } \\ \text { bomba-ABS.PL } & \text { longo-INS } & \text { explodir-repetidamente-IND.[-tr].3PL } \\ \text { 'Bombas explodiram repetidamente } & \text { por um longo tempo.' }\end{array}$

(VAN GEENHOVEN, 2005) 
L. SANCHEZ-

Mendes, R. R.

Segala \& A.

N. XAVIER

O papel da (re)

duplicação na

expressão de

pluracionalidade

em libras

Já em dyirbal (pama-nyungan), a reduplicação parcial no verbo está relacionada a uma semântica de intensidade, conforme ilustrado abaixo.
a. balgan
'golpear'
Dyirbal
b. balbalgan
'golpear muito'

(LASERSOHN, 1995)

Estudos recentes sobre a pluracionalidade mostram que nem todas as línguas expressam todas as leituras atestadas translinguisticamente. Além disso, eles também evidenciam que dentro de uma mesma língua é possível encontrar condições semânticas que podem estar envolvidas na seleção das leituras associadas aos marcadores pluracionais.

Em checheno (caucasiana), por exemplo, a telicidade determina a leitura expressa pela pluracionalidade. Grosso modo, predicados télicos são aqueles que denotam eventos que têm um fim determinado lexicalmente, como 'chegar' e 'atravessar a rua'. Predicados atélicos, por sua vez, denotam eventos que não possuem um fim determinado pelo léxico, como 'correr' e 'desenhar círculos' (VENDLER, 1957). Em checheno, quando predicados télicos como 'atirar' recebem o marcador pluracional, que tem a forma de alternância vocálica, a leitura da sentença é de pluralidade de eventos.
(10) a. as q'iigashna twop-qwessira. Checheno 1sG corvo.PL.DAT arma-atirar.WP
'Eu atirei nos corvos.'
$\begin{array}{llll}\text { b. } & \text { as } & \text { q'iigashna } & \text { twop-qissira. } \\ & \text { 1sG } & \text { corvo.PL.DAT } & \text { arma-atirar.PLR.wP }\end{array}$

'Eu atirei nos corvos muitas vezes.'

Por outro lado, quando a sentença apresenta um predicado atélico, a leitura desencadeada pela pluracionalidade é de duração do evento, conforme ilustrado abaixo com o verbo 'doer'.
(11)
Cyna
chow
xoizhira.
Checheno
3sG.Poss
ferida
doer.wP
'Sua ferida doeu (momentaneamente).'
b. Cyna chow xiizhira.
3sg.poss ferida doer.PLR.wP
'Sua ferida doeu (por um longo tempo).'

(YU, 2003)

Revista Letras,

Curitiba, UFPR,

n. 96, pp.487-508,

jul./dez. 2017.

ISSN 2236-0999

(versão eletrônica) 
O conjunto de dados reunidos nesta seção mostra tanto a diversidade morfofonológica quanto semântica da pluracionalidade. Esses dados contribuem para a adoção de uma ampla perspectiva quando da análise desse fenômeno em qualquer língua. Ou seja, é preciso levar em conta que a pluracionalidade é um fenômeno semântico que pode se manifestar das mais diferentes formas e ter uma gama de leituras como efeito de significado. Essa foi a perspectiva adotada na análise dos dados da libras que será apresentada neste artigo.

\section{Pluracionalidade em Línguas de Sinais}

O objetivo desta seção é apresentar brevemente a análise de SanchezMendes e Xavier (2016) para a pluracionalidade em libras, em razão de seus dados serem reanalisados neste artigo. Além disso, apresentaremos também a proposta de Kuhn e Aristodemo (2017) para a pluracionalidade em língua de sinais francesa (LSF), da qual partiu nossa investigação para a repetição dos sinais em libras.

Sanchez-Mendes e Xavier (2016) investigaram o efeito semântico da duplicação de mãos de verbos produzidos tipicamente com uma mão. Para isso, analisaram 14 de 28 sinais (listados em 12) elicitados por Xavier (2014) de surdos usuários de libras. Embora investigados de uma perspectiva fonético-fonológica, esses dados foram preliminarmente descritos pelo autor como casos que tiveram

494 sua duplicação de mãos motivada por fatores semânticos.

$$
\begin{aligned}
& \text { ACUSAR, AVISAR, BEIJAR, IGNORAR, PAGAR, } \\
& \text { VER, APRENDER, COLAR, COMER, ENTENDER, } \\
& \text { IMAGINAR, INVENTAR, IR-EMBORA, RIR }
\end{aligned}
$$

A análise desses fatores semânticos foi refinada por Sanchez-Mendes e Xavier (2016) que mostram que os significados expressos pela duplicação de mãos se referem a plural de argumentos, plural de eventos e intensidade. A proposta defendida pelos autores é a de que todos esses efeitos semânticos expressos podem ser abarcados pela mesma operação semântica, a pluracionalidade, apontando para a semelhança desse fenômeno em muitas línguas orais de diversos lugares do mundo.

Ademais, eles mostraram que a direcionalidade dos verbos é um fator que determina as leituras apresentadas pela duplicação. Verbos direcionais são aqueles cuja forma varia de acordo com a posição no espaço de sinalização dos participantes a que seus argumentos fazem referência (FELIPE DE SOUZA, 1998). Segundo Sanchez-Mendes e Xavier (2016), quando verbos monomanuais dessa classe têm o seu número de mãos duplicado, eles apresentam leitura de 
L. SANCHEZ-

Mendes, R. R.

Segala \& A.

N. XAVIER

O papel da (re)

duplicação na

expressão de

pluracionalidade

em libras

plural de argumentos. Os verbos não direcionais, por sua vez, não restringem as leituras e apresentam interpretações de plural de argumentos, de plural de eventos e de intensidade. Os exemplos abaixo ilustram um caso de cada leitura. Em (13), há um exemplo com o verbo AVISAR em que a duplicação de mãos expressa plural de argumentos. Em (14), o verbo APRENDER apresenta a leitura de plural de eventos quando é produzido com duas mãos. Por fim, em (15), o verbo RIR apresenta leitura de intensidade.

(13) Duplicação de mãos do verbo AVISAR

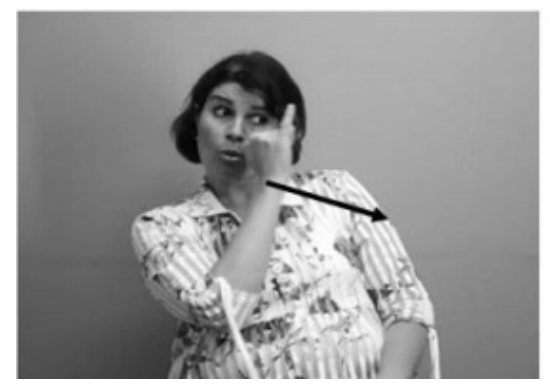

'Eu o(a) aviso(ei).'

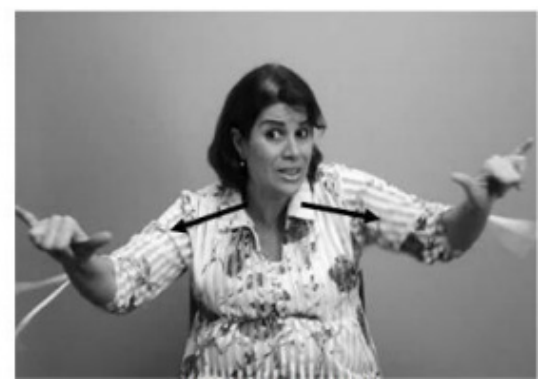

'Eu os(as) aviso(ei).'

(14) Duplicação de mãos do verbo APRENDER
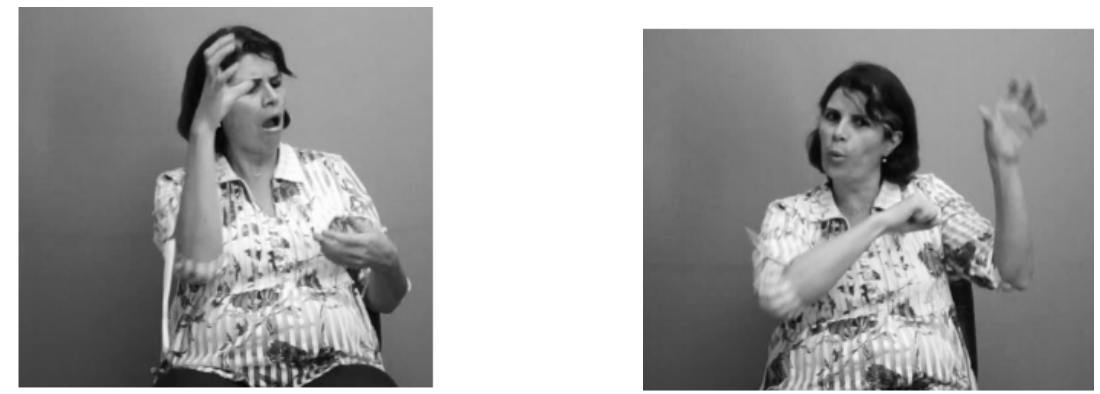

'Aprendi sobre a psicanálise.

'Na segunda aprendi sobre passe, na terça....fui aprendendo ao longo da semana.'

(15) Duplicação de mãos do verbo RIR

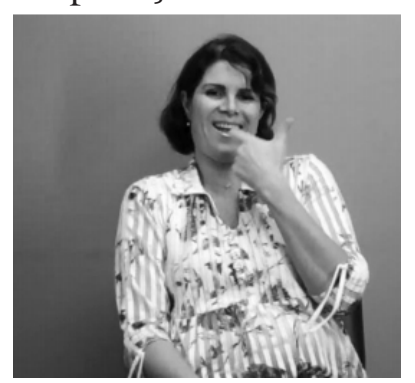

'Rir (normal)'

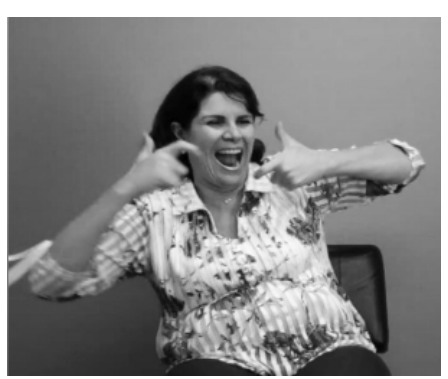

'Rir muito'

(SANCHEZ-MENDES; XAVIER, 2016)

Vale dizer que essas leituras foram atestadas nesse conjunto específico de dados. A generalização em Sanchez-Mendes e Xavier (2016) deve ser testada

Revista Letras,

Curitiba, UFPR, n. 96, pp.487-508, jul./dez. 2017. ISSN 2236-0999 (versão eletrônica) 
em um conjunto de dados maior. Além disso, conforme apontado pelos autores, é possível que haja restrições semânticas entre os verbos não-direcionais que selecionem as leituras. Neste ponto da pesquisa ainda não é possível afirmar que restrições são essas ou quais verbos podem apresentar mais de uma das leituras.

Kuhn e Aristodemo (2017), por sua vez, investigaram a expressão de pluracionalidade na língua de sinais francesa (LSF). Em seu estudo, os autores focaram em duas formas verbais. Em uma delas, o verbo, em sua forma monomanual, é repetido em sequência (REP). Em outra, o verbo aparece em sua forma bimanual, sendo repetido por meio de movimentos alternados (ALT). O exemplo (16) ilustra essas duas formas por meio do sinal ESQUECER.

(16) Formas do sinal ESQUECER, reproduzidas de Kuhn e Aristodemo (2017)

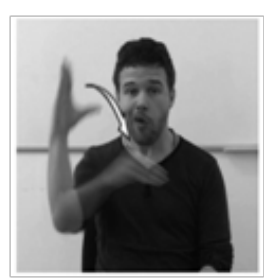

'ESQUECER'

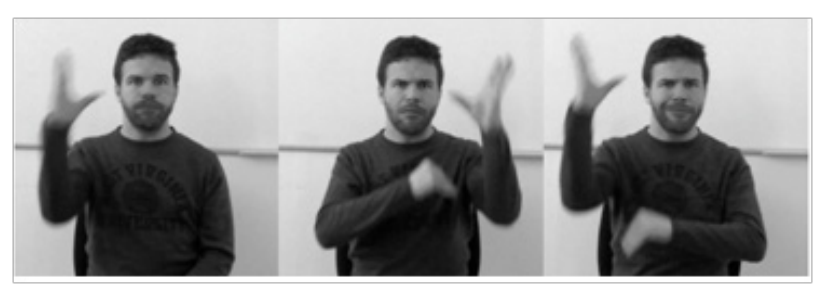

'ESQUECER-REP'

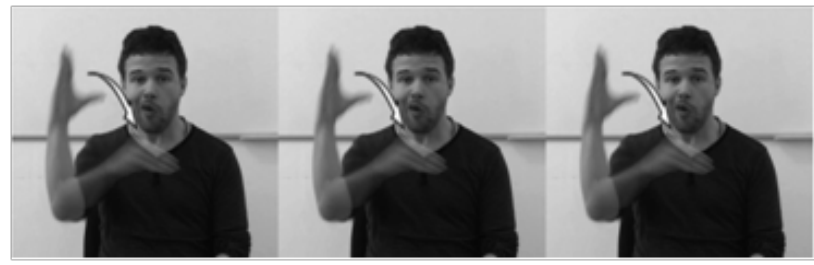

'ESQUECER-ALT'

De acordo com Kuhn e Aristodemo, na LSF, essa diferença na forma é empregada na LSF para marcar uma diferença semântica. Embora em ambos os casos, a repetição do sinal expresse plural de evento, em (17a), todos os eventos denotados pelo verbo estão associados a um mesmo participante. Diferentemente, em (17b), cada evento de esquecer está associado a mais de um participante.

\section{a. JEAN CÂMERA TRAZER ESQUECER-rep.} 'Jean esquece de trazer a câmera repetidamente.'

b. AMIGO POSS-1 IX-arc CÂMERA TRAZER ESQUECER-alt. 'Cada um dos meus amigos esqueceu de trazer a câmera.'

(KUHN, ARISTODEMO, 2017)

Vale notar que, em LSF, a repetição do verbo com movimento alternado pode ocorrer sempre que um dos participantes da sentença seja plural. Em (17b) acima, ele foi utilizado com sujeito plural. Em (18a) abaixo, a mesma forma foi utilizada com objeto plural. Essa ocorrência só será agramatical quando não houver nenhum participante plural na sentença, conforme ilustrado em (18b). 
L. SANCHEZ-

Mendes, R. R.

Segala \& A.

N. Xavier

O papel da (re)

duplicação na

expressão de

pluracionalidade em libras
(18)

\author{
a. UMA PESSOA ESQUECEU-ALt MUITAS PALAVRAS. \\ 'Uma pessoa esqueceu muitas palavras.' \\ b. „JEANCHEGOU-ALt. \\ Intenção: 'Jean chegou.'
}

(KUHN, ARISTODEMO, 2017)

Os dados da LSF apresentam um fator diferente para a comparação entre formas mono e bimanuais expressando pluracionalidade nas línguas de sinais: a forma com que o evento é repetido, se de forma alternada ou não. Embora os autores não deixem claro se esperam que esse comportamento na LSF seja universal nas línguas de sinais, o presente trabalho tem como objetivo avançar na descrição da expressão de pluracionalidade em libras verificando se a mesma generalização observada para a LSF pode ser feita para a libras.

\section{Metodologia}

Os dados discutidos neste trabalho provêm, primeiramente, de uma reanálise interpretativa dos 14 analisados por Sanchez-Mendes e Xavier (2016) e, em seguida, de uma nova eliciação desses mesmos sinais, objetivando: (a) verificar a possibilidade de serem realizados repetidamente com uma mão e com duas mãos movendo-se alternadamente e (b) identificar as leituras associadas a essas realizações. Ambos os procedimentos envolveram o mesmo sujeito surdo (sinalizante nativo de libras, com 37 anos, da cidade de São Paulo e com pósgraduação) e tiveram o intuito, respectivamente, de (i) corroborar a análise desses mesmos dados apresentados por Sanchez-Mendes e Xavier (2016) e (ii) checar se a libras, em relação aos dados em questão, apresenta comportamento semelhante ao documentado por Kuhn e Aristodemo (2017) para a LSF.

Primeiramente, a reanálise interpretativa consistiu em solicitar ao sujeito surdo o exame dos vídeos, coletados por meio de elicitação por Xavier (2014), contendo as formas mono e bimanual dos 14 sinais listados em (19) em contexto. Depois de assistir a cada vídeo, foi solicitado ao referido sujeito que explicasse, com base em sua intuição, a ocorrência da duplicação de mãos naqueles casos. Apesar da natureza metalinguística da tarefa solicitada, nada foi dito acerca da análise das leituras previamente identificadas para as formas realizadas com duas mãos.

(19) ACUSAR, APRENDER, AVISAR, BEIJAR, COLAR (na prova), COMER, ENTENDER, IGNORAR, IMAGINAR, INVENTAR, IR-EMBORA, PAGAR, RIR, VER.

Revista Letras,

Curitiba, UFPR, n. 96, pp.487-508,

jul./dez. 2017. ISSN 2236-0999 (versão eletrônica) 
Os dados obtidos através da reanálise interpretativa foram capturados em vídeo pelo próprio sujeito surdo em sua residência e na ausência dos demais pesquisadores. Essa fase da coleta foi importante porque ela corroborou a análise apresentada em Sanchez-Mendes e Xavier (2016).

Já a segunda etapa da coleta consistiu em duas etapas: (i) teste de gramaticalidade, ou seja, se cada um dos sinais em (19) pode ser realizado repetidamente e em sequência tanto na sua forma monomanual repetida quanto na sua forma bimanual com movimento alternado; e (ii) teste de interpretação de sentenças, em que foi inquirido qual o significado que esses sinais veiculariam em cada caso. Mais especificamente, testamos se as formas repetidas sem e com alternância poderiam fazer referência a um único participante e/ou a mais de um.

Nessafase inicial,nãoseparamosos verbossegundoonúmerodeargumentos nem direcionamos a pergunta para participantes específicos do evento. Para cada verbo a pergunta enfocava todos os seus participantes, independentemente de sua função sintática ou papel temático. Refinar essas noções é uma das próximas etapas desta pesquisa que poderá sugerir qual o comportamento da libras em relação ao alinhamento de seus argumentos (nominativo ou ergativo) bem como estabelecer possíveis restrições semânticas para a pluralização.

Os dados obtidos através da nova eliciação foram também capturados em vídeos, mas em dois momentos diferentes. No primeiro deles, os três autores deste trabalho, dois ouvintes e um surdo, se encontraram virtualmente via videoconferência (Skype). Ao autor ouvinte bilíngue, coube a função de intermediar as perguntas realizadas pela outra autora ouvinte, que também ficou responsável por tomar nota das respostas do autor surdo. O autor surdo teve a liberdade de expressar suas intuições quando se sentiu pronto durante toda a sessão. Toda a reunião foi capturada em vídeo por meio do software webnaria ${ }^{2}$ (Figura 1).

2 http://www.webinaria.com/ 


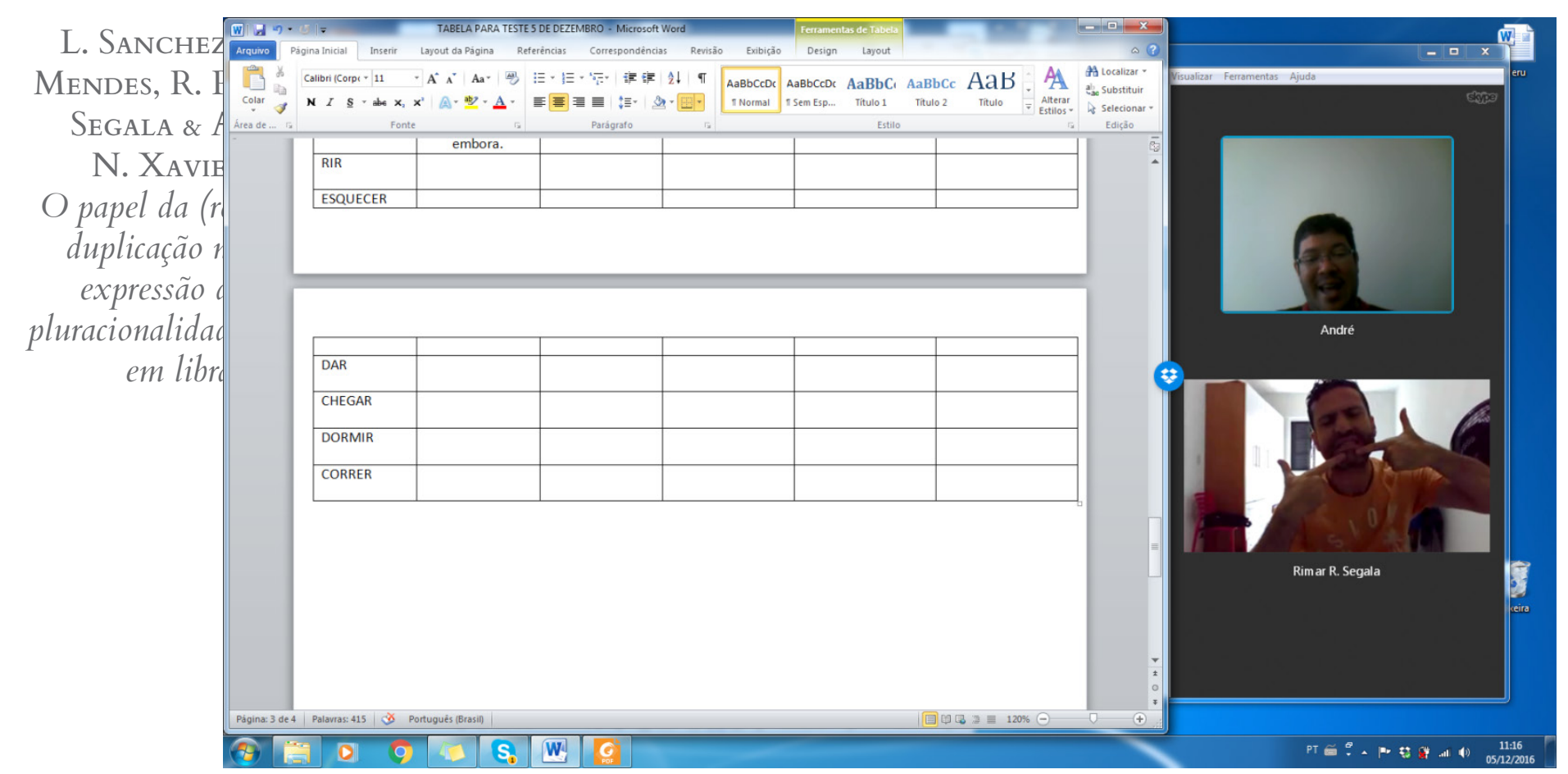

Figura (1). Print da tela da pesquisadora ouvinte durante a reunião via vídeo conferência

O uso da videoconferência enquanto ferramenta metodológica revelouse bastante adequada para casos em que autores e/ou colaboradores se encontram em cidades diferentes. Além disso, a coleta com a participação de três pessoas (um colaborador, um pesquisador fazendo as perguntas e um terceiro elaborando perguntas e tomando notas) foi muito produtiva.

O segundo momento envolveu a solução de dúvidas adicionais que surgiram durante a análise dos dados e foram respondidas por vídeos enviados pelo autor surdo por meio de mensagens de vídeo (via Whatsapp). O uso de aparatos tecnológicos tais como videoconferência e mensagens de vídeo auxiliam a dinâmica de pesquisas em que nem sempre é possível reunir pessoalmente todos os pesquisadores envolvidos.

\section{Resultados}

A reanálise interpretativa, por um sinalizante surdo nativo de libras, de 14 dos 28 sinais coletados por Xavier (2014) apontou duas leituras para a maioria das formas produzidas com duas mãos: pluralidade e intensidade. De acordo com a intuição do referido sinalizante, expressa pluralidade a forma bimanual dos sinais em (20) e intensidade a dos sinais em (21). 

$\operatorname{VER}(2)^{4}$.

DIRECIONAIS

\author{
APRENDER, COMER, IMAGINAR, INVENTAR, PAGAR(2), \\ RIR.
}

Em relação à análise de Sanchez-Mendes e Xavier (2016), podemos observar que os resultados aqui reportados a corroboram de duas formas. Primeiramente, porque reiteram as leituras que, segundo os autores, estão associadas à pluracionalidade em libras: pluralidade e intensidade. ${ }^{5}$ Em segundo lugar, porque foi confirmado que a forma bimanual de todos os verbos direcionais (20b) foi interpretada como expressando pluralidade, conforme a descrição anterior.

Cabe registrar, no entanto, que houve pequenas discrepâncias em termos da leitura atribuída à forma bimanual de alguns sinais. Apesar disso, elas não invalidam a análise apresentada em Sanchez-Mendes e Xavier (2016), mas apenas apontam para a complexidade de trabalhar com a dissociação entre as leituras expressas pela operação de pluracionalidade. Enquanto para o sinalizante surdo nativo de libras todos os sinais em (21) expressam intensidade, para Sanchez-Mendes e Xavier (2016) isso se daria apenas com o sinal RIR. De acordo com os autores, a forma bimanual dos demais expressa pluralidade: de eventos no caso de APRENDER e IMAGINAR e de argumentos no caso de COMER, INVENTAR e PAGAR. Tal fato deve resultar da grande dificuldade de se dissociar, em muitos casos, leituras de plural de evento e plural de argumentos de leituras de intensidade. Em um dos exemplos analisado pelo sinalizante, a forma bimanual com movimento alternado é empregada quando a sinalizante se refere a alguém que, mesmo depois de comer, vai em busca de mais comida e come várias coisas sem parar (exemplo 22). Assim, para alguns sinalizantes essa leitura pode ser descrita como plural de argumentos (comer várias coisas) ou como intensidade (comer muito).

$$
\text { Exemplo de uso do sinal COMER em sua versão bimanual }
$$

\footnotetext{
3 O sinalizante surdo nativo interpretou alguns exemplos de uso de um mesmo sinal produzidos por diferentes colaboradas do estudo de Xavier (2014) de forma diferente. Nesses casos, indicou-se com o número 1 ou 2 o exemplo de qual delas se está fazendo menção. Quando a interpretação foi idêntica para os dois exemplos, nenhuma notação foi utilizada.

4 O sinalizante surdo nativo deixou explícita sua dúvida quanto à interpretação da forma bimanual de VER com movimento reto como mais um caso de expressão de pluralidade.

5 Embora não seja trivial separar as leituras de pluralidade e intensidade nas línguas, estamos mantendo essa separação. Considera-se plural sempre que há dois ou mais eventos ou participantes envolvidos, e a intensidade está sendo tratada em seu sentido estrito, como uma intensificação de alguma propriedade associado ao evento denotado pelo verbo.
} 
L. SANCHEZ-

Mendes, R. R.

Segala \& A.

N. Xavier

O papel da (re)

duplicação na

expressão de

pluracionalidade

em libras

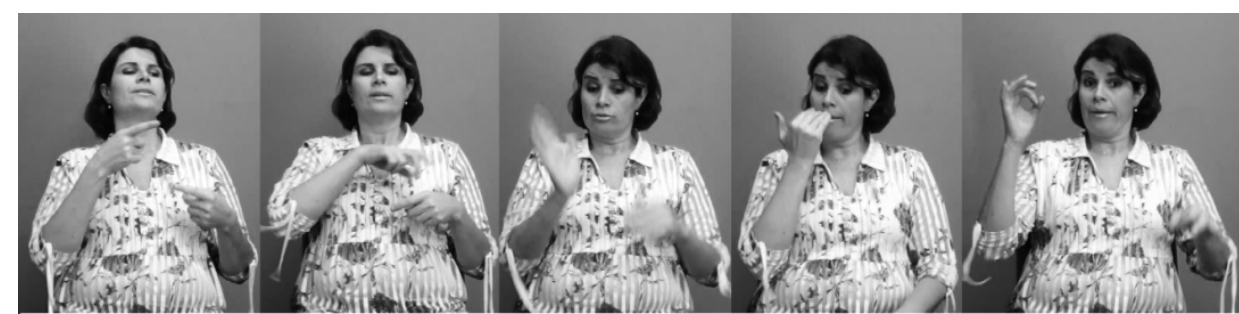

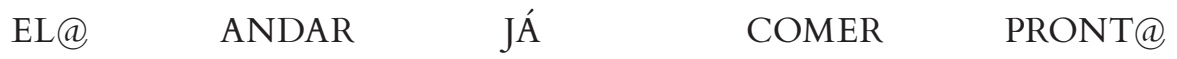

'Ele foi (até um certo lugar) e comeu'

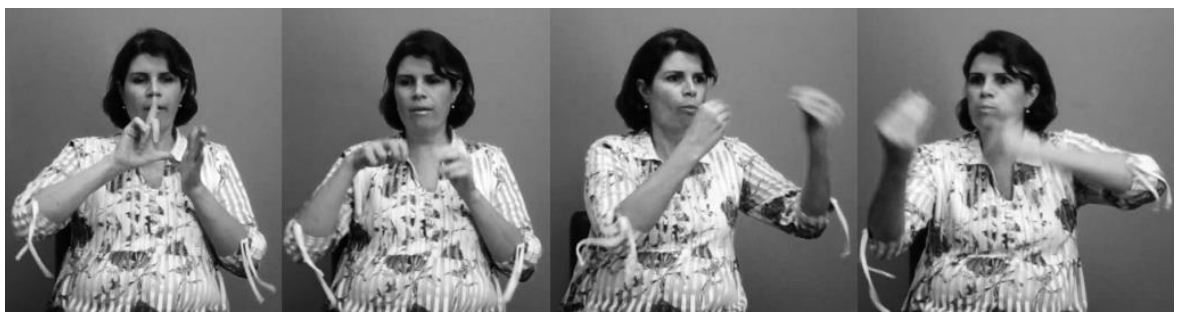

DEPOIS

ANDAR

COMER

'Depois, foi (até o mesmo lugar) e comeu, comeu, comeu'

Um outro ponto de desacordo entre os dados coletados e reportados neste artigo e os publicados em Sanchez-Mendes e Xavier (2016) diz respeito ao uso da forma bimanual do sinal AVISAR e do sinal ENTENDER por uma das colaboradoras surdas do estudo de Xavier (2014), aqui designada como colaboradora 2, e do sinal VER por ambas as colaboradoras do mesmo estudo. Quando produziu com duas mãos o sinal AVISAR, a colaboradora 2 empregou movimento circular, diferentemente da colaboradora 1, que o produziu com movimento reto. Já quando realizou com duas mãos o sinal ENTENDER, a colaboradora 2 focou seu olhar numa mesma direção, diferentemente da colaboradora 1, que moveu sua cabeça de um lado para o outro, dirigindo seu olhar para vários pontos à sua frente. Por fim, quando ambas as colaboradoras produziram a versão bimanual de VER, elas empregaram movimento circular alternado.

Embora Sanchez-Mendes e Xavier (2016) tenham tratado os exemplos de uso envolvendo AVISAR e VER como casos de expressão de pluralidade de argumentos e o exemplo envolvendo ENTENDER como intensidade, o sinalizante surdo nativo os interpretou como desviantes dos padrões semânticos identificados anteriormente. A justificativa para isso se vinculou ao fato de que a forma bimanual de AVISAR com movimento circular tem um significado relacionado, mas diferente daquele que se observa quando o mesmo sinal é produzido com movimento reto. Segundo ele, essa forma não significa simplesmente avisar a mais de uma pessoa, mas já adquiriu um significado mais especializado que envolve a noção de avisar uma coletividade e, portanto, deve ser traduzida como 'divulgar' em português. Já no que diz respeito a ENTENDER, o referido sinalizante diz que a forma lhe parece estranha e que não se recorda de
Revista Letras,

Curitiba, UFPR, n. 96, pp.487-508,

jul./dez. 2017. ISSN 2236-0999 (versão eletrônica) 
tê-la usado ou visto sendo usada. Por fim, em relação à forma bimanual de VER, produzida pela colaboradora 1 com movimento circular alternado, o sinalizante nativo a interpretou como independente do sinal VER, já que não conseguiu associá-lo nem à expressão de pluralidade, nem à de intensidade.

Esses resultados mostram, primeiramente, que a duplicação de mãos pode ser utilizada como um recurso derivacional de formação de novas palavras. Esse é o caso de AVISAR que passa a expressar DIVULGAR quando é produzido com duas mãos e movimento circular. Esse fenômeno não é exclusivo da libras. Lasersohn (1995) em seu trabalho acerca da pluracionalidade em língua orais salienta a natureza derivacional dessa operação. Ou seja, não é de se espantar que em alguns casos o verbo pluracional passe a ter um uso mais especializado.

O que os outros exemplos apontam é que há um outro fator morfofonológico que ainda não foi bem explorado na análise desses dados: a diferença entre fazer o movimento de forma reta ou de forma circular. O desacordo entre os dados coletados pode ter ocorrido por essa diferença. Isso aponta que esse é um fator a ser explorado em pesquisas futuras, bem como outras condições, tais como direção do olhar e movimento da cabeça.

A análise dos dados coletados através da nova eliciação dos sinais primeiramente analisados por Xavier (2014) revelou que a repetição em sequência da forma monomanual é possível para os todos os sinais. O sinalizante surdo nativo fez uma única ressalva em relação ao verbo INVENTAR que, de acordo com sua intuição, parece ser pouco frequente nessa forma. A repetição da forma bimanual por meio de movimentos alternados das mãos, no entanto, apesar de possível para a maioria dos sinais, causou ao referido sinalizante um certo estranhamento quando aplicada aos sinais ENTENDER e IGNORAR. Isso pode ter ocorrido por razões semânticas. ENTENDER e IGNORAR parecem se comportar como verbos psicológicos típicos. Ao contrário dos eventos que podem ser individuados, pluralizados e distribuídos no tempo, verbos psicológicos denotam estados que são verdadeiros a respeito de um sujeito em momentos de tempo determinados, mas não estão relacionados a uma ação executada que progride no tempo. Dessa forma, eles têm mais resistência em ser pluralizados (VENDLER, 1957).

Já no que diz respeito ao significado, a análise mostrou que tanto a forma mono quanto a bimanual com movimento repetido são empregadas para se fazer referência à pluralidade de eventos. A diferença entre a repetição com uma mão e a repetição alternada espelha a encontrada nos exemplos da LSF observada por Kuhn e Aristemo (2017) na LSF. Ou seja, todas as produções de repetição do sinal com uma mão estão relacionadas a uma iteração do evento no tempo e todas as formas repetidas alternadas envolvem situações com participantes plurais.

A única exceção foi, novamente, o sinal ENTENDER, cuja forma monomanual com repetição de movimento, expressa, segundo a intuição do sinalizante surdo nativo, esforço. Esse resultado pode estar associado ao fato 
L. SANCHEZ-

Mendes, R. R.

Segala \& A.

N. XAVIER

O papel da (re)

duplicação na

expressão de

pluracionalidade

em libras

de se tratar de um verbo psicológico que pode ter uma leitura de intensidade traduzida como esforço. Esses resultados são sumarizados na tabela 1 e ilustrados no exemplo (23).

\begin{tabular}{|l|c|c|}
\hline \multicolumn{1}{|c|}{ Verbos } & $\begin{array}{c}\text { Repetição } \\
\text { da forma } \\
\text { monomanual }\end{array}$ & $\begin{array}{c}\text { Repetição da forma } \\
\text { bimanual com movimento } \\
\text { alternado }\end{array}$ \\
\hline $\begin{array}{l}\text { ACUSAR, APRENDER, } \\
\text { AVISAR, BEIJA, COLAR (na } \\
\text { prova), COMER, ENTENDER, } \\
\text { IMAGINAR, INVENTAR, IR- } \\
\text { EMBORA, PAGAR, RIR, VER. }\end{array}$ & $\checkmark$ & $\checkmark$ \\
\hline $\begin{array}{l}\text { ENTENDER } \\
\text { IGNORAR }\end{array}$ & $\checkmark$ & \\
\hline
\end{tabular}

Tabela 1. Resultados da eliciação

Sinal COLAR em suas formas de repetição monomanual e bimanual com alternância

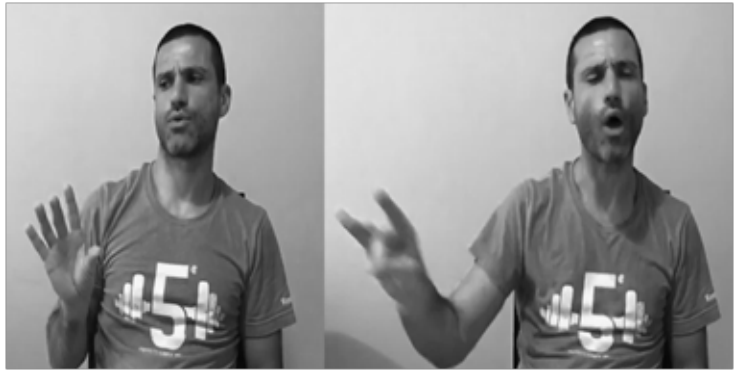

'Colar várias vezes de uma pessoa'

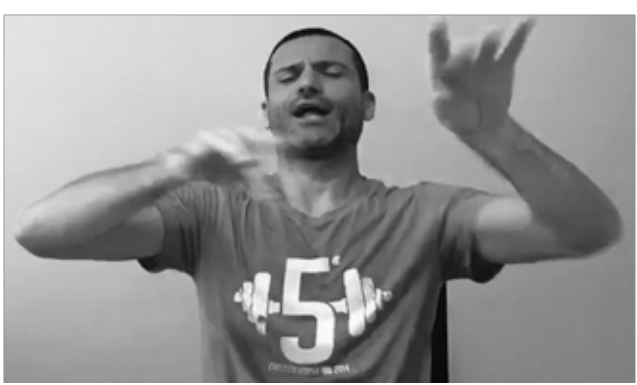

'Todos colando'

\section{Proposta Formal}

Nesta seção, apresentamos nossa proposta formal para a análise da pluracionalidade em libras. Vamos argumentar que, a despeito da variedade de leituras atestadas, a pluracionalidade em libras envolve uma operação de plural (cf. LASERSOHN, 1995; YU 2003; MÜLLER, SANCHEZ-MENDES, 2010; entre outros) e, de forma mais geral, como uma operação sobre uma variável escalar que pode ser cardinalidade ou intensidade.

Assim, a entrada lexical básica (provisória) da pluracionalidade em libras é apresentada em (23). Em outras palavras, (23) expressa que a operação de plural se aplica a um predicado de eventos $P$ e devolve um predicado cujos eventos não sejam atômicos, ou seja, um predicado plural.

$$
\llbracket \mathrm{Pl} \rrbracket=\lambda \mathrm{P} \lambda \mathrm{e} \cdot \mathrm{P}(\mathrm{e}) \wedge \text { não-atômico }(\mathrm{e})
$$


A partir da operação básica de plural, é possível atribuir uma semântica mais específica para os casos de leitura especializada, como as que vimos nos dados de repetição monomanual em comparação com repetição bimanual com alternância do movimento. Para os casos com leitura iterativa como a expressa pela repetição do sinal com uma mão, adiciona-se à denotação a informação de que os tempos das ocorrências dos eventos não sejam iguais. Ou seja, cada um dos sub-eventos de $e$ ocorrem em tempos diferentes.

$$
\begin{aligned}
& \llbracket \mathrm{Pl} \text { REP } \rrbracket=\lambda \mathrm{P} \lambda \text { e. } \mathrm{P}(\mathrm{e}) \wedge \text { não-atômico }(\mathrm{e}) \wedge \$ \mathrm{e}^{\prime}, \mathrm{e}^{\prime} \leq \mathrm{e}\left[\mathrm{\tau}\left(\mathrm{e}^{\prime}\right) \neq\right. \\
& \left.\tau\left(\mathrm{e}^{\prime \prime}\right)\right]
\end{aligned}
$$

A fórmula acima pode ser lida da seguinte forma: a pluracionalidade expressa por meio de repetição é uma operação que pega um predicado verbal $P$ e devolve um predicado de eventos $e$ que seja plural (não-atômico); o conjunto de eventos possui sub-eventos $e^{\prime}$ e $e$ " que são parte de $e$ e que possuem tempos de ocorrência $\tau$ diferentes.

Da mesma forma, é possível fornecer uma semântica mais específica para a pluracionalidade expressa por meio da repetição do sinal com alternância das mãos. Vimos que, nesse caso, a leitura é a de que houve um plural de argumentos. Assim sendo, acrescenta-se à denotação a informação de que os papeis temáticos atribuídos a cada um dos sub-eventos de e sejam diferentes, garantindo a existência de participantes diferentes para cada sub-evento.

$$
\begin{aligned}
& \llbracket \mathrm{Pl} \text { ALt } \rrbracket=\lambda \mathrm{P} \lambda \text { e. } \mathrm{P}(\mathrm{e}) \wedge \text { não-atômico }(\mathrm{e}) \wedge \$ \mathrm{e}^{\prime}, \mathrm{e}^{\prime \prime} \leq \mathrm{e}\left[\theta\left(\mathrm{e}^{\prime}\right) \neq\right. \\
& \left.\theta\left(\mathrm{e}^{\prime \prime}\right)\right]
\end{aligned}
$$

A fórmula acima pode ser lida da seguinte forma: a pluracionalidade expressa por meio de repetição alternada é uma operação que pega um predicado verbal $P$ e devolve um predicado de eventos $e$ que seja plural (não-atômico); o conjunto de eventos possui sub-eventos $e^{\prime}$ e $e$ " que são parte de $e$ e que possuem papeis temáticos $\theta$ diferentes.

As diferenças entre as denotações em (24) e (25) está na escolha do critério de individuação do evento plural, se por tempo de ocorrência ou por participantes. Dessa forma, vemos que a diferença morfofonológica entre simples repetição do sinal e a repetição com alternância indica a seleção da dimensão sobre a qual o evento plural será distribuído, se sobre tempos de ocorrência (repetição) ou sobre participantes (alternância). Dessa forma fica clara qual é a contribuição semântica de cada uma das manifestações morfofonológicas: a (re)duplicação indica que a existência de um predicado de eventos plurais, enquanto que a distinção entre repetição e alternância indica o critério de individuação desses eventos.

Um dos desafios para a análise da pluracionalidade como operação básica de plural está na captura da leitura de intensidade tal como a que foi apresentada 
L. SANCHEZ-

Mendes, R. R.

Segala \& A.

N. XAVIER

O papel da (re)

duplicação na

expressão de

pluracionalidade

em libras

para o verbo RIR, por exemplo. Não é trivial obter essa leitura apenas adicionando uma informação sobre a dimensão em que o evento é distribuído, tal como foi feito em (24) e (25).

Nossa proposta preliminar para a tentativa de unificação de análise para os casos em que há plural (de eventos e argumentos) e intensidade é considerar que estamos lidando com duas escalas diferentes, uma que avalia o predicado segundo a cardinalidade de seus eventos e outra que o avalia segundo a sua intensidade. Dessa forma, é possível considerar que a pluracionalidade lida com essas duas dimensões escalares ${ }^{6}$. Vamos utilizar uma variável sobre dimensões $\mu$ (tal como proposta em Krifka (1998), Nakanishi (2007), Thomas (2009) e Sanchez-Mendes (2014)). Essa variável pode ser substituída por uma dimensão de cardinalidade nos casos de leitura de plural de eventos (distribuídos sobre tempo de ocorrências ou sobre participantes) (26a) ou por um sintagma de medida que mede a intensidade no caso da leitura de intensidade (26b).

$$
\begin{array}{ll} 
& \llbracket \mathrm{Pl} \rrbracket=\lambda \mathrm{P} \lambda \text { e. } \mathrm{P}(\mathrm{e}) \wedge \mu(\mathrm{e}) \geq \mathrm{n} \\
\text { a. } & \llbracket \mathrm{Pl} \rrbracket=\lambda \mathrm{P} \lambda \text { e. } \mathrm{P}(\mathrm{e}) \wedge \operatorname{card}(\mathrm{e}) \geq \mathrm{n} \\
\text { b. } & \llbracket \mathrm{Pl} \rrbracket=\lambda \mathrm{P} \lambda \text { e. } \mathrm{P}(\mathrm{e}) \wedge \mathrm{SM}_{-} \mathrm{INT}(\mathrm{e}) \geq \mathrm{n}
\end{array}
$$

Nossa proposta de formalização avança em comparação à oferecida para a LSF por Kuhn e Aristodemo (2017) porque trata da leitura de intensidade, ainda que preliminarmente, além das leituras que envolvem plural. De toda forma, a proposta apresentada nesta seção é apenas uma tentativa de formalização das leituras encontradas com a expressão da pluracionalidade em libras. Nessa altura da pesquisa ainda não é possível prever com quais tipos de verbos cada uma das leituras vai emergir e qual das fórmulas, portanto, deve ser utilizada em cada caso. Conforme discutido mais abaixo, esse é um dos próximos passo da pesquisa.

\section{Considerações finais}

Este artigo mostrou que o mecanismo morfofonológico geral de (re) duplicação expressa pluracionalidade em libras. Defendemos que todas as leituras associadas à pluracionalidade podem ser capturadas por meio de uma operação básica sobre o predicado que pode ser avaliado segundo duas dimensões, uma de cardinalidade, que avalia os eventos plurais, e uma de intensidade. Dentre as leituras plurais, mostramos que os diferentes tipos de reduplicação estão associados a diferentes critérios de individuação dos eventos, se sobre tempos de ocorrência ou sobre participantes.

6 Não vamos nos aprofundar na semântica de escalas. Para uma visão geral dos mecanismos de graus e escalas, ver Kennedy $(1999,2007)$ e Kennedy e McNally (2005).

Revista Letras,

Curitiba, UFPR,

n. 96, pp.487-508,

jul./dez. 2017.

ISSN 2236-0999

(versão eletrônica) 
Os próximos passos da pesquisa envolvem análise mais aprofundada dos diferentes tipos de movimento, reto e circular, das expressões faciais e corporais e do uso do espaço averiguando mais uma vez qual é a contribuição semântica de cada um dos aspectos morfofonológicos dos verbos da libras. Além disso, pretende-se investigar de que forma as classes verbais podem estar envolvidas na seleção das diferentes leituras expressas pela operação de pluracionalidade. De forma mais geral, essa investigação poderá mostrar de que forma o fenômeno da pluracionalidade pode contribuir para a análise das classes verbais da libras, tema bem pouco explorado. 
L. SANCheZ-

Mendes, R. R.

Segala \& A.

N. Xavier

O papel da (re)

duplicação na

expressão de

pluracionalidade

em libras

\section{Referências}

BAR-EL, L. Verbal number and aspect in Skwxwú7mesh. Recherches linguistiques de Vincennes, v. 37, p. 31-54, 2008.

CABREDO-HOFHERR, P.; LACA, B. Verbal Plurality and Distributivity. Berlin: de Gruyter, 2012.

COLLINS, C. Aspects of plurality in thoan. Language, v. 77, n. 3, p. 456-476, 2001.

CUSIC, D. D. Verbal Plurality and Aspect. Tese (Doutorado em Linguística), Stanford University, Stanford, 1981.

FELIPE DE SOUZA, T. A. A relação sintático-semântica dos verbos e seus argumentos na lingua brasileira de sinais (libras). 1998. Tese (Doutorado em Linguística) Universidade Federal do Rio de Janeiro, Rio de Janeiro, 1998.

GIL, D. From repetition to reduplication in Riau Indonesian. In: HURCH, B. (Ed.). Studies on Reduplication: Empirical Approaches to Language Typology 28. Berlin: Mouton de Gruyter, 2005. p. 31-64.

KENNEDY, C. Projecting the adjective: The syntax and semantics of gradability and comparison. New York: Garland. 1999.

Vagueness and Grammar: The Semantics of Relative and Absolute Gradable Adjectives. Linguistics and Philosophy 30.1, 2007.

KENNEDY, C.; MCNALLY, L. Scale Structure, Degree Modification, and the Semantics of Gradable Predicates. Language, v. 81, n. 2, p. 345-381, 2005.

KRIFKA, M. The origins of telicity. In: ROTHSTEIN, S. (Ed.) Events and grammar. Dordrecht: Kluwer, 1998. p. 197-235.

KUHN, J.; ARISTODEMO, V. Pluractionality, iconicity, and scope in French Sign Language. Semantics and Pragmatics, v. 10, n. 6, 2017.

LASERSOHN, P. Plurality, Conjunction, and Events. Boston: Kluwer Academic Publishers, 1995.

MÜLLER, A.; SANCHEZ-MENDES, L. O Significado da Pluracionalidade em Karitiana. Cadernos de Estudos Linguísticos, Campinas, v. 52, n. 2, p. 215231, 2010.

NAKANISHI, K. Formal Properties of Measurement Constructions. Berlin: Mouton de Gruyter, 2007.

RUBINO, C. Reduplication: Form, function and distribution. In: HURCH, B. (Ed.). Studies on Reduplication: Empirical Approaches to Language Typology 28. Berlin: Mouton de Gruyter, 2005. p. 11-29.

Revista Letras,

Curitiba, UFPR,

n. 96, pp.487-508,

jul./dez. 2017.

ISSN 2236-0999

(versão eletrônica) 
SANCHEZ-MENDES, L. A Modificação de Grau em Karitiana. 2014. Dissertação (Mestrado em Linguística) - Universidade Federal do Rio de Janeiro, Rio de Janeiro, 2014.

SANCHEZ-MENDES, L.; XAVIER, A. N. A expressão da pluracionalidade em Libras. Revista Estudos Linguísticos, São Paulo, v. 45, n. 1, p. 292-304, 2016.

THOMAS. G. Comparison across domains in Mbyá. Proceedings of WSCLA 14. Vancouver: Department of Linguistics, University of British Columbia, 2009.

VAN GEENHOVEN, V. Atelicity, Pluractionality and Quantification In: VERKUYL, H.; DE SWART, H. (Eds.) Perspectives on Aspect. Dordrecht: Springer, 2005. p. 107-124.

VENDLER, Z. Verbs and times. The Philosophical Review, v. 66 n. 2, p. 143160, 1957.

YU, A. Pluractionality in Chechen. Natural Language Semantics, v. 11, p. 289321, 2003. XAVIER, A. N. Uma ou duas? Eis a questão! Um estudo do parâmetro número de mãos na produção de sinais da língua brasileira de sinais (libras). 2014. Tese (Doutorado em Linguística). Instituto de Estudos da Linguagem, Universidade Estadual de Campinas, Campinas, 2014.

Submetido em: 05/03/2017

Aceito em: 03/08/2017 